\title{
Threshold resummation of transverse momentum distributions beyond next-to-leading log
}

\author{
Stefano Forte, ${ }^{a}$ Giovanni Ridolfi ${ }^{b}$ and Simone Rota ${ }^{a}$ \\ ${ }^{a}$ Tif Lab, Dipartimento di Fisica, Università di Milano and \\ INFN, Sezione di Milano, \\ Via Celoria 16, I-20133 Milano, Italy \\ ${ }^{b}$ Dipartimento di Fisica, Università di Genova and \\ INFN, Sezione di Genova, \\ Via Dodecaneso 33, I-16146 Genova, Italy \\ E-mail: forte@mi.infn.it, giovanni.ridolgi@ge.infn.it, \\ simone.rota@unimi.it
}

ABSTRACT: We derive a general expression for the threshold resummation of transverse momentum distributions for processes with a colorless final state, by suitably generalizing the renormalization-group based approach to threshold resummation previously pursued by two of us. The ensuing expression holds to all logarithmic orders, and it can be used to extend available results in the literature, which only hold up to the next-to-leading log (NLL) level. We check agreement of our result with the existing NLL result, as well as against the known fixed next-to-leading order results for the Higgs transverse momentum distribution in gluon fusion, and we provide explicit expressions at the next-to-next-toleading log level.

Keywords: Resummation, Perturbative QCD

ArXiv EPrint: 2106.11321 


\section{Contents}

1 Introduction 1

2 Phase space factorization $\quad 2$

2.1 Kinematics 2

2.2 Phase space 4

3 Resummation $\quad 8$

3.1 The renormalization group argument with a single scale 8

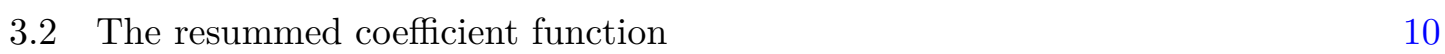

$\begin{array}{ll}3.3 & \text { Two-scale resummation and the transverse momentum distribution }\end{array}$

4 Resummed results $\quad 13$

$\begin{array}{lll}4.1 & \text { Next-to-leading resummation } & 13\end{array}$

$\begin{array}{ll}4.2 \text { Comparison to fixed-order calculation } & 14\end{array}$

5 Outlook 15

$\begin{array}{ll}\text { A Explicit resummed results } & 16\end{array}$

$\begin{array}{ll}\text { B The NLO transverse momentum distribution } & 19\end{array}$

\section{Introduction}

Sudakov resummation is routinely used to improve the domain of validity and the perturbative convergence of fixed-order calculations of differential observables at the LHC. For example, the transverse momentum distribution of Higgs and gauge bosons produced in hadronic collisions has been recently determined at matched $\mathrm{NNLO}+\mathrm{N}^{3} \mathrm{LL}$, i.e., fixed next-to-next-to-leading-order and resummed next ${ }^{3}$-leading-logarithmic order in transversemomentum logarithms [1-4]. However, threshold resummation is typically not included in the computation of differential observables, despite the fact that recent work has shown that combining threshold on top of transverse momentum resummation has a significant impact on perturbative convergence $[5,6]$. This is especially surprising in view of the common use of threshold resummation to improve the perturbative convergence of total cross-sections [7]. In fact, it turns out that threshold resummation for transverse momentum distributions is currently only available in direct QCD up to next-to leading log (NLL) [8], with NNLL results available only in SCET $[9,10]$

As pointed out in ref. [8], threshold resummation for transverse momentum distributions is structurally similar to - and can be viewed as a generalization of - the soft resummation of the total cross-section for prompt-photon production [11] to which it may 
be related using the general resummation formalism for multiparton processes of ref. [12]. A general approach to soft resummation based on renormalization group (RG) arguments was proposed by some of us long ago [13], based on the earlier approach of ref. [14], and shortly thereafter extended to include the case of prompt-photon production [15]. This approach has the advantage of generality: it provides the form of resummed results to any logarithmic order, though it does not allow for the computation of the coefficients that determine the resummed expressions explicitly, which must be found by matching to fixed-order expressions - the fixed $\mathrm{N}^{k} \mathrm{LO}$ fully determines the $\mathrm{N}^{k} \mathrm{LL}$ resummed result.

Here, we extend the approach of ref. [13] to transverse momentum distributions, by viewing them as a generalization of the case of prompt-photon production [15]. We arrive at a form of the soft resummation which is in fact somewhat more compact than that of ref. [8], with which we prove agreement at NLL. We work out the general form of the resummed expressions up to NNLL. We check the agreement with fixed NLO result for Higgs production in gluon fusion [16], which verifies the correctness of the resummed result up to NLL. This comparison was already performed numerically in ref. [8]; here we show that the resummation correctly predicts the logarithmically enhanced terms in the fixedorder result. A check of the NNLL result would require comparing the NNLO fixed order. While for Higgs production in gluon fusion this is principle available [17, 18], in practice the explicit analytic expression which would be needed in order to read off the relevant coefficient is not readily obtained.

The generalization of the RG argument of ref. [15] to transverse-momentum distributions requires an analysis of the phase space structure for real emission in the soft limit that will be presented in section 2 . This will be used in section 3 to derive the general form of resummed results, that in section 4 will be compared to available results.

\section{Phase space factorization}

We consider the transverse momentum distribution $\frac{d \sigma}{d p_{\mathrm{T}}^{2}}$ for the process

$$
H_{1}\left(P_{1}\right)+H_{2}\left(P_{2}\right) \rightarrow H+X
$$

where $H$ is a final-state object (particle or system), whose invariant mass will be denoted by $m$, and $p_{\mathrm{T}}$ is the transverse momentum of $H$ with respect to the direction of the colliding hadrons $H_{1}$ and $H_{2}$.

\section{$2.1 \quad$ Kinematics}

The transverse momentum distribution is characterized by two scales, which can be constructed out of the invariant mass $m$ and transverse momentum $p_{\mathrm{T}}$, and a scaling variable $\tau$. Factorization follows if the scaling variable is chosen as

$$
\tau \equiv \frac{\left(E_{\mathrm{T}}+p_{\mathrm{T}}\right)^{2}}{s},
$$


where $s=\left(P_{1}+P_{2}\right)^{2}$ and we denote with $p_{\mathrm{T}}$ the modulus of the transverse momentum vector and with $E_{\mathrm{T}}$ the transverse energy

$$
E_{\mathrm{T}}=\sqrt{m^{2}+p_{\mathrm{T}}^{2}} ; \quad p_{\mathrm{T}}=\left|\vec{p}_{\mathrm{T}}\right| .
$$

This suggests the choice of

$$
Q=\sqrt{m^{2}+p_{\mathrm{T}}^{2}}+\sqrt{p_{\mathrm{T}}^{2}}=E_{\mathrm{T}}+p_{\mathrm{T}}
$$

as one of the two scales of the process, so that

$$
\tau=\frac{Q^{2}}{s}
$$

As we shall see below, a natural choice for the other independent scale is $Q p_{\mathrm{T}}$ : indeed this was denoted as $Q^{2}$ in ref. [8], and we shall therefore consider henceforth the cross-section as a function of $Q^{2}$ and $Q p_{\mathrm{T}}$.

The scale $Q$ eq. (2.4) is the threshold energy, i.e. the minimum energy needed to produce a system with invariant mass $m$ and transverse momentum $p_{\mathrm{T}}$. This ensures factorization in that, with this choice, the kinematic boundary for the scaling variable $\tau$ at fixed $p_{\mathrm{T}}$ is $p_{\mathrm{T}}$-independent:

$$
0 \leq \tau \leq 1
$$

so

$$
\frac{d \sigma}{d p_{\mathrm{T}}^{2}}\left(\tau, Q^{2}, Q p_{\mathrm{T}}\right)=\tau \sum_{i, j} \int_{\tau}^{1} \frac{d x}{x} \mathcal{L}_{i j}\left(\frac{\tau}{x}\right) \frac{1}{x} \frac{d \hat{\sigma}_{i j}}{d p_{\mathrm{T}}^{2}}\left(x, Q^{2}, Q p_{\mathrm{T}}\right)
$$

where

$$
x=\frac{Q^{2}}{\hat{s}}=\frac{\left(\sqrt{m^{2}+p_{\mathrm{T}}^{2}}+p_{\mathrm{T}}\right)^{2}}{\hat{s}},
$$

$\hat{s}$ is the center-of-mass partonic energy squared, and the parton luminosity is defined in the usual way as

$$
\mathcal{L}_{i j}(x)=\int_{x}^{1} \frac{d y}{y} f_{i}(y) f_{j}\left(\frac{x}{y}\right) .
$$

To keep notations simple, the dependence of the quantities involved on the strong coupling $\alpha_{s}\left(\mu_{\mathrm{R}}^{2}\right)$ and on the factorization scale $\mu_{\mathrm{F}}$ are omitted in this section.

Because of the $p_{\mathrm{T}}$ independence of the limits of integration, eq. (2.7) factorizes upon taking a Mellin transform with respect to the scaling variable $\tau$. Namely, defining

$$
\begin{aligned}
\frac{d \sigma}{d p_{\mathrm{T}}^{2}}\left(N, Q^{2}, Q p_{\mathrm{T}}\right) & =\int_{0}^{1} d \tau \tau^{N-1} \frac{d \sigma}{d p_{\mathrm{T}}^{2}}\left(\tau, Q^{2}, Q p_{\mathrm{T}}\right) ; \\
\frac{d \hat{\sigma}_{i j}}{d p_{\mathrm{T}}^{2}}\left(N, Q^{2}, Q p_{\mathrm{T}}\right) & =\int_{0}^{1} d x x^{N-1} \frac{d \hat{\sigma}_{i j}}{d p_{\mathrm{T}}^{2}}\left(x, Q^{2}, Q p_{\mathrm{T}}\right),
\end{aligned}
$$

where with a slight abuse of notation we are using the same symbol for the cross section and its Mellin transform, we get

$$
\frac{d \sigma}{d p_{\mathrm{T}}^{2}}\left(N, Q^{2}, Q p_{\mathrm{T}}\right)=\sum_{i j} \mathcal{L}_{i j}\left(N+1, \mu_{\mathrm{F}}^{2}\right) \frac{d \hat{\sigma}_{i j}}{d p_{\mathrm{T}}^{2}}\left(N, Q^{2}, Q p_{\mathrm{T}}\right) .
$$


Note that because the scale $Q$ eq. (2.4), and consequently the scaling variable eq. (2.5) depend on $p_{\mathrm{T}}$, they necessarily differ from the scale and scaling variable used for the total cross-section.

It is also useful to introduce the variable

$$
\bar{Q} \equiv E_{\mathrm{T}}-p_{\mathrm{T}},
$$

so that

$$
p_{\mathrm{T}}=\frac{1}{2}(Q-\bar{Q})
$$

and

$$
E_{\mathrm{T}}=\frac{1}{2}(Q+\bar{Q}) .
$$

The definition eq. (2.13) implies that

$$
Q \bar{Q}=E_{\mathrm{T}}^{2}-p_{\mathrm{T}}^{2}=m^{2}
$$

or

$$
\bar{Q}=\frac{m^{2}}{Q} .
$$

It follows in particular that substituting the expression eq. (2.17) in the expression eq. (2.14) of $p_{\mathrm{T}}$ we get

$$
Q p_{\mathrm{T}}=\frac{Q^{2}-m^{2}}{2},
$$

which shows that any pair of variables among $Q p_{\mathrm{T}}, Q^{2}, m^{2}$ can be chosen as independent kinematic variables, along with the dimensionless ratio $\tau$ eq. (2.5). With any such choice there are two scales and a scaling variable, which can be varied independently without conflicting with factorization, i.e. in such a way that at the factorized level the parton luminosity only depends on $\tau$ and a scale.

\subsection{Phase space}

We now consider the phase space measure for the process eq. (2.1) in the soft limit. We will follow the treatment of ref. [15], which in turn exploits the general formalism for dealing with an $n$-body phase-space discussed in the appendix of ref. [13], recently generalized, systematized, and supplemented with a graphical representation in ref. [19].

Specifically, we consider a contribution to the transverse momentum distribution in which there are $k+1$ massless partons in the final state, with momenta $k_{1}, \ldots, k_{k+1}$, and

$$
p_{1}+p_{2}=p_{H}+k_{1}+\ldots+k_{k+1} .
$$

We are interested in the threshold limit. For a $p_{\mathrm{T}}$ distribution eq. (2.1), there must be at least a non-soft final state parton in $X$ that recoils against $H$. Hence, the threshold limit can be approached when all other partons are either collinear to this non-soft parton, or soft. We then assume that momenta $k_{i}, i=1, \ldots, n ; n \leq k$ are soft, while momenta $k_{i}, i>n$ are non-soft. For the sake of simplicity, we relabel non-soft momenta as

$$
k_{j}^{\prime}=k_{n+j} ; \quad 1 \leq j \leq m+1 ; \quad m=k-n .
$$


The generic kinematic configuration in the soft limit is then

$$
\begin{array}{rlrl}
k_{i} & =0 & 1 \leq i \leq n & \\
\theta_{i j} & =0 ; & \sum_{j=1}^{m+1} k_{j}^{\prime 0}=p_{\mathrm{T}} & 1 \leq i, j \leq m+1
\end{array}
$$

$\left(\theta_{i j}\right.$ being the angle formed by $\vec{k}_{i}$ and $\left.\vec{k}_{j}\right)$ for all $n$ between 1 and $k$, namely, the configuration where at least one momentum is not soft, and the remaining momenta are either collinear to it, or soft.

With this labeling of the momenta, the phase space can be written as

$$
\begin{aligned}
& d \phi_{n+m+2}\left(p_{1}+p_{2} ; p_{H}, k_{1}, \ldots, k_{n}, k_{1}^{\prime}, \ldots, k_{m+1}^{\prime}\right) \\
& =\int \frac{d q^{2}}{2 \pi} d \phi_{n+1}\left(p_{1}+p_{2} ; q, k_{1}, \ldots, k_{n}\right) \int \frac{d k^{\prime 2}}{2 \pi} d \phi_{2}\left(q ; p_{H}, k^{\prime}\right) d \phi_{m+1}\left(k^{\prime} ; k_{1}^{\prime}, \ldots, k_{m+1}^{\prime}\right) .
\end{aligned}
$$

Here:

- $d \phi_{n+1}$ is the inclusive phase space for a production process with two incoming partons with momenta $p_{1}, p_{2}$, of a massive object with mass $q^{2}$, plus $n$ partons with momenta $k_{i}$ (to be taken as soft).

- $d \phi_{2}$ is the phase space for production, from an incoming momentum $q$, of a massive final state with mass $m$, whose momentum $p_{H}$ will be assumed to have a fixed transverse momentum $p_{\mathrm{T}}$, and a system with momentum $k^{\prime}$ recoiling against it. Note that in the soft limit in which all momenta $k_{i}$ vanish, $q$ reduces to $p_{1}+p_{2}$, so $q^{2}=s$.

- $d \phi_{m+1}$ is the phase space for the production, from incoming momentum $k^{\prime}$, of a finalstate system containing $m+1$ partons with momenta $k_{i}^{\prime}$. Note that in the soft limit, in which all momenta $k_{i}$ vanish, and all momenta $k_{i}^{\prime}$ are collinear to $k^{\prime}$, momenta $p_{H}$ and $k^{\prime}$ are back-to-back. Note also that in the small $p_{\mathrm{T}}$ limit all momenta $k^{\prime}$ are also collinear to the incoming parton's direction (but not soft).

We first work out the two-body phase space $d \phi_{2}$. In $4-2 \epsilon$ dimensions and in the rest frame of $q$ we have

$$
\begin{aligned}
d \phi_{2}\left(q ; p_{H}, k^{\prime}\right) & =\frac{d^{d-1} k^{\prime}}{(2 \pi)^{d-1} 2 k^{\prime 0}} \frac{d^{d-1} p_{H}}{(2 \pi)^{d-1} 2 p_{H}^{0}}(2 \pi)^{d} \delta^{(d)}\left(q-k^{\prime}-p_{H}\right) \\
& =\frac{(4 \pi)^{\epsilon}}{16 \pi \Gamma(1-\epsilon)} p_{\mathrm{T}}^{-2 \epsilon} \frac{d p_{\mathrm{T}}^{2} d p_{z}}{k_{0}^{\prime} p_{H}^{0}} \delta\left(p_{H}^{0}+k_{0}^{\prime}-\sqrt{q^{2}}\right) .
\end{aligned}
$$

The delta can be used to perform the integration in $p_{z}$. In the rest frame of $q$ we find

$$
\delta\left(p_{H}^{0}+k_{0}^{\prime}-\sqrt{q^{2}}\right)=\frac{\delta\left(p_{z}-\bar{p}_{z}\right)}{\left|J\left(\bar{p}_{z}\right)\right|}
$$

where

$$
\bar{p}_{z}^{2}=\frac{\lambda\left(m^{2}, q^{2}, k^{2}\right)}{4 q^{2}}-p_{\mathrm{T}}^{2} ; \quad \lambda(x, y, z)=x^{2}+y^{2}+z^{2}-2 x y-2 x z-2 y z
$$


and

$$
\left|J\left(\bar{p}_{z}\right)\right|=\bar{p}_{z}\left(\frac{1}{p_{H}^{0}}+\frac{1}{k_{0}^{\prime}}\right)=\frac{\bar{p}_{z} \sqrt{q^{2}}}{p_{H}^{0} k_{0}^{\prime}} .
$$

Hence

$$
d \phi_{2}\left(q ; p_{H}, k^{\prime}\right)=\frac{(4 \pi)^{\epsilon}}{16 \pi \Gamma(1-\epsilon)} \frac{p_{\mathrm{T}}^{-2 \epsilon}}{\bar{p}_{z} \sqrt{q^{2}}} d p_{\mathrm{T}}^{2} .
$$

Next, we work out the kinematic limits for the integration variables $q^{2}$ and $k^{\prime 2}$. We have

$$
\begin{aligned}
p_{1}+p_{2} & =q+\sum_{i=1}^{n} k_{i} \\
q & =p_{H}+k^{\prime} .
\end{aligned}
$$

We start with the bounds for $q^{2}$. Equation (2.28) implies $q^{2} \leq \hat{s}=\left(p_{1}+p_{2}\right)^{2}$. Eq. (2.29) implies, in the rest frame of $q$, which in the soft limit coincides with the center-of-mass frame,

$$
q^{2}=\left(\sqrt{m^{2}+p_{\mathrm{T}}^{2}+p_{z}^{2}}+\sqrt{{k^{\prime}}^{2}+p_{\mathrm{T}}^{2}+p_{z}^{2}}\right)^{2}
$$

so the minimum value of $q^{2}$ is attained when ${k^{\prime}}^{2}=p_{z}=0$, and it is equal to $q_{\min }^{2}=Q^{2}$. Hence we conclude that

$$
Q^{2} \leq q^{2} \leq \hat{s}
$$

We consider next the bounds on ${k^{\prime}}^{2}$. Equation (2.29) implies

$$
k^{\prime 2}=q^{2}+m^{2}-2 \sqrt{q^{2}} \sqrt{m^{2}+p_{\mathrm{T}}^{2}+p_{z}^{2}}
$$

so the maximum value of $k^{\prime}$ is attained when $p_{z}=0$ :

$$
\begin{aligned}
k_{\max }^{\prime 2} & =q^{2}+m^{2}-2 \sqrt{q^{2}} E_{\mathrm{T}} \\
& =q^{2}+m^{2}-\sqrt{q^{2}}\left(Q+\frac{m^{2}}{Q}\right),
\end{aligned}
$$

where in the last step we have used eqs. (2.15), (2.17).

We can finally write the full phase space as follows:

$$
\begin{aligned}
& d \phi_{n+m+2}\left(p_{1}+p_{2} ; p_{H}, k_{1}, \ldots, k_{n}, k_{1}^{\prime}, \ldots, k_{m+1}^{\prime}\right)=\frac{(4 \pi)^{\epsilon}}{64 \pi^{3} \Gamma(1-\epsilon)} p_{\mathrm{T}}^{-2 \epsilon} d p_{\mathrm{T}}^{2} \\
& \quad \times \int_{Q^{2}}^{s} \frac{d q^{2}}{\sqrt{q^{2}}} \int_{0}^{k_{\text {max }}^{\prime 2}} \frac{d{k^{\prime}}^{2}}{\bar{p}_{z}} d \phi_{n+1}\left(p_{1}+p_{2} ; q, k_{1}, \ldots, k_{n}\right) d \phi_{m+1}\left(k^{\prime} ; k_{1}^{\prime}, \ldots, k_{m+1}^{\prime}\right) .
\end{aligned}
$$

We now consider the soft limit, $x \rightarrow 1$. We introduce a dimensionless parameter $0 \leq u \leq 1$ interpolating between the two extremes for $q^{2}$. We have

$$
q^{2}=Q^{2}+u\left(\hat{s}-Q^{2}\right)=Q^{2}\left(1+u \frac{1-x}{x}\right)
$$

which shows that $q^{2} \rightarrow Q^{2}$ in the soft limit. 
Using eq. (2.35) to express $q^{2}$ in terms of the independent kinematic variables we get

$$
\begin{aligned}
k_{\max }^{\prime 2} & =Q^{2}\left(1+u \frac{1-x}{x}\right)+m^{2}-\left(Q^{2}+m^{2}\right) \sqrt{1+u \frac{1-x}{x}} \\
& =\frac{1}{2}\left(Q^{2}-m^{2}\right) u(1-x)+O\left((1-x)^{2}\right) \\
& =Q p_{\mathrm{T}} u(1-x)+O\left((1-x)^{2}\right),
\end{aligned}
$$

where we have used eq. (2.18) in the last step. Introducing a further dimensionless parameter $0 \leq v \leq 1$ in order to interpolate between the two extremes we have

$$
k^{\prime 2}=u v Q p_{\mathrm{T}}(1-x)
$$

Hence in the soft limit ${k^{\prime}}^{2} \rightarrow 0$.

Finally, in this limit,

$$
q^{2} \bar{p}_{z}^{2}=p_{\mathrm{T}} E_{\mathrm{T}} Q^{2}(1-x) u(1-v)[1+O(1-x)]
$$

and therefore

$$
\begin{aligned}
& d \phi_{n+m+2}\left(p_{1}+p_{2} ; p_{H}, k_{1}, \ldots, k_{n}, k_{1}^{\prime}, \ldots, k_{m+1}^{\prime}\right) \\
& =\frac{(4 \pi)^{\epsilon}}{64 \pi^{3} \Gamma(1-\epsilon)} p_{\mathrm{T}}^{-2 \epsilon} d p_{\mathrm{T}}^{2} Q^{2}(1-x)^{3 / 2} \sqrt{\frac{p_{\mathrm{T}}}{E_{\mathrm{T}}}} \int_{0}^{1} d u \int_{0}^{1} d v \sqrt{\frac{u}{1-v}} \\
& \quad d \phi_{n+1}\left(p_{1}+p_{2} ; q, k_{1}, \ldots, k_{n}\right) d \phi_{m+1}\left(k^{\prime} ; k_{1}^{\prime}, \ldots, k_{m+1}^{\prime}\right) .
\end{aligned}
$$

The full phase space thus factors into the product of two phase spaces, related by the $u$ and $v$ integrations, with $p_{\mathrm{T}}$ kept fixed.

Phase space $d \phi_{n+1}\left(p_{1}+p_{2} ; q, k_{1}, \ldots, k_{n}\right)$ is the same as that for Drell-Yan or Higgs production, namely for the production of a colorless final state of mass $q^{2}$, as given in ref. [13] (see in particular eq. (4.30) of that reference). Note that it does not depend on $p_{\mathrm{T}}$. In the soft limit, this phase space can be written in terms of a dimensionless integration measure, with all the dimensional dependence contained in a prefactor, given by a power of

$$
\frac{\left(\hat{s}-q^{2}\right)^{2}}{q^{2}} \propto Q^{2}(1-x)^{2} \equiv \Lambda_{\mathrm{DY}}^{2}
$$

Phase space $d \phi_{m+1}\left(k^{\prime} ; k_{1}^{\prime}, \ldots, k_{m+1}^{\prime}\right)$ can be viewed as a phase space with the same structure of deep-inelastic scattering, namely incoming momentum $k^{\prime 2}$, with the variable $k^{\prime}$ now integrated over, and vanishing in the soft limit, again as given in ref. [13] (see in particular eq. (4.17) of that reference). This too can be written in terms of a dimensionless integration measure, with now the dimensional dependence contained in a power of

$$
k^{\prime 2} \propto Q p_{\mathrm{T}}(1-x) \equiv \Lambda_{\mathrm{DIS}}^{2}
$$

In summary, in the soft limit the phase space for transverse momentum distributions factorizes completely into a Drell-Yan-like phase space, related to soft emission, that only depends on the dimensional scale $\Lambda_{\mathrm{DY}}^{2}$ eq. (2.40), and a DIS-like phase space, related to collinear emission, that only depends on the dimensional scale $\Lambda_{\text {DIS }}^{2}$ eq. (2.41). Note that what determines the scale is not whether emission is from incoming or outgoing legs, but rather, whether the emission can contribute in the soft limit because it is soft, or because it is collinear to the fixed $p_{\mathrm{T}}$ parton that recoils against the fixed- $p_{\mathrm{T}}$ final state $H$. 


\section{Resummation}

The resummation argument is a rerun of that of ref. [15], which generalizes to promptphoton production the resummation approach developed and discussed in refs. [13, 14], specifically for deep-inelastic scattering (DIS) and Drell-Yan (DY) production. The basic underlying idea remains the same, but in processes like DIS and DY one single soft scale is resummed, while in prompt photon production, as well as in the case of transverse momentum distributions discussed here, two different soft scales are simultaneously resummed. We first briefly summarize the argument of refs. [13-15] in a somewhat generalized form, and then we use it to obtain a resummed expression for transverse momentum distributions exploiting the results presented in the previous section.

\subsection{The renormalization group argument with a single scale}

Resummation is most easily expressed for a coefficient function, which is defined factoring out of the cross-section the Born-level expression. Specifically, for a total cross-section

$$
\hat{\sigma}_{i j}\left(N, Q^{2}, \alpha_{s}\left(Q^{2}\right)\right)=C_{i j}\left(N, Q^{2} / \mu^{2}, \alpha_{s}\left(\mu^{2}\right)\right) \sigma_{i j}^{0}\left(N, Q^{2}, \mu^{2}, \alpha_{s}\left(\mu^{2}\right)\right)
$$

where $\sigma_{i j}^{0}$ is the leading-order expression, and we have chosen for simplicity $\mu_{F}^{2}=\mu_{R}^{2}=\mu^{2}$. In the soft limit, only diagonal partonic channels are unsuppressed, resummation can be performed independently in the quark singlet and gluon channel and we will consequently suppress the parton indices $i, j$.

We discuss first the case of a process with a single hard scale [13, 14], such as DY or DIS. Resummation is performed in terms of the physical anomalous dimension

$$
\gamma\left(N, Q^{2} / \mu^{2}, \alpha_{s}\left(\mu^{2}\right), \epsilon\right)=\frac{d}{d \ln Q^{2}} \ln C\left(N, Q^{2} / \mu^{2}, \alpha_{s}\left(\mu^{2}\right), \epsilon\right),
$$

where we have adopted dimensional regularization with $4-2 \epsilon$ space-time dimensions. The coefficient function is multiplicatively renormalized: in a mass-independent subtraction scheme,

$$
C\left(N, Q^{2} / \mu^{2}, \alpha_{s}\left(\mu^{2}\right), \epsilon\right)=Z^{C}\left(N, \alpha_{s}\left(\mu^{2}\right), \epsilon\right) C^{(0)}\left(N, Q^{2}, \alpha_{0}, \epsilon\right),
$$

where $C^{(0)}$ and $\alpha_{0}$ are the bare coefficient function and coupling respectively. It follows that the physical anomalous dimension can be equivalently computed from the bare coefficient function [13]:

$$
\gamma\left(N, Q^{2} / \mu^{2}, \alpha_{s}\left(\mu^{2}\right), \epsilon\right)=-\epsilon \alpha_{0} \frac{d}{d \ln \alpha_{0}} \ln C^{(0)}\left(N, Q^{2}, \alpha_{0}, \epsilon\right),
$$

where we have used the fact that $C^{(0)}$ can only depend on $Q^{2}$ and $\alpha_{0}$ through the dimensionless combination $Q^{-2 \epsilon} \alpha_{0}$. For a single-scale process in the soft limit the dimensional dependence of the phase space is through a fixed combination of the scale and the scaling variable

$$
\Lambda_{a}^{2}\left(x, \lambda^{2}\right)=\lambda^{2}(1-x)^{a},
$$


where $a=1$ in the case of DIS, and $a=2$ in the case of DY. This implies [13] that the Mellin-space coefficient function only depends on $N$ through the dimensional variable

$$
\bar{\Lambda}_{a}^{2}\left(N, \lambda^{2}\right)=\frac{\lambda^{2}}{N^{a}}
$$

Assuming further full factorization of the soft singularities [14], this in turn implies that the coefficient function admits a perturbative expansion of the form

$$
\begin{aligned}
C^{(0)}\left(N, Q^{2}, \alpha_{0}, \epsilon\right) & =C^{(0, c)}\left(Q^{2}, \alpha_{0}, \epsilon\right) C^{(0, l)}\left(\bar{\Lambda}_{a}^{2}\left(N, Q^{2}\right), \alpha_{0}, \epsilon\right) \\
C^{(0, c)}\left(Q^{2}, \alpha_{0}, \epsilon\right) & =\sum_{n} C_{n}^{(0, c)}(\epsilon) Q^{-2 n \epsilon} \alpha_{0}^{n} \\
C^{(0, l)}\left(\bar{\Lambda}^{2}, \alpha_{0}, \epsilon\right) & =\sum_{n} C_{n}^{(0, l)}(\epsilon) \bar{\Lambda}^{-2 n \epsilon} \alpha_{0}^{n}
\end{aligned}
$$

where $C^{(0, l)}$ collects contributions due to real emission, which have nontrivial kinematics, $C^{(0, c)}$ collects virtual contributions, that have Born kinematics, and factorization is the assumption that virtual (or "hard") and real soft-emission contributions fully factorize.

Equation (3.7) implies the decomposition of the physical anomalous dimension

$$
\gamma\left(N, \frac{Q^{2}}{\mu^{2}}, \alpha_{s}\left(\mu^{2}\right), \epsilon\right)=\gamma^{(c)}\left(\frac{Q^{2}}{\mu^{2}}, \alpha_{s}\left(\mu^{2}\right), \epsilon\right)+\gamma^{(l)}\left(\frac{\bar{\Lambda}_{a}^{2}\left(N, Q^{2}\right)}{\mu^{2}}, \alpha_{s}\left(\mu^{2}\right), \epsilon\right)
$$

where

$$
\begin{aligned}
\gamma^{(c)}\left(\frac{Q^{2}}{\mu^{2}}, \alpha_{s}\left(\mu^{2}\right), \epsilon\right) & =\epsilon \alpha_{0} \frac{d}{d \ln \alpha_{0}} \ln C^{(0, c)}\left(Q^{2}, \alpha_{0}, \epsilon\right) \\
\gamma^{(l)}\left(\frac{\bar{\Lambda}_{a}^{2}\left(N, Q^{2}\right)}{\mu^{2}}, \alpha_{s}\left(\mu^{2}\right), \epsilon\right) & =\epsilon \alpha_{0} \frac{d}{d \ln \alpha_{0}} \ln C^{(0, l)}\left(\bar{\Lambda}_{a}^{2}\left(N, Q^{2}\right), \alpha_{0}, \epsilon\right) .
\end{aligned}
$$

The two contributions $\gamma^{(c)}$ and $\gamma^{(l)}$ are not necessarily separately finite, and thus depend a priori on the scale $\mu$. However, their sum, the physical anomalous dimension $\gamma$, is finite and renormalization-group invariant, so

$$
-\frac{d}{d \ln \mu^{2}} \lim _{\epsilon \rightarrow 0} \gamma^{(l)}\left(\frac{\bar{\Lambda}_{a}^{2}}{\mu^{2}}, \alpha_{s}\left(\mu^{2}\right), \epsilon\right)=\frac{d}{d \ln \mu^{2}} \lim _{\epsilon \rightarrow 0} \gamma^{(c)}\left(\frac{Q^{2}}{\mu^{2}}, \alpha_{s}\left(\mu^{2}\right), \epsilon\right)=\bar{g}\left(\alpha_{s}\left(\mu^{2}\right)\right)
$$

where $\bar{g}\left(\alpha_{s}\left(\mu^{2}\right)\right.$ is a perturbative function of $\alpha_{s}$ with finite coefficients:

$$
\bar{g}\left(\alpha_{s}\left(\mu^{2}\right)\right)=\sum_{n} \bar{g}_{n} \alpha_{s}^{n}\left(\mu^{2}\right)
$$

Equation (3.13) can be viewed as a standard renormalization-group equation for the physical anomalous dimension, with solution

$$
\gamma\left(N, 1, \alpha_{s}\left(Q^{2}\right), \epsilon\right)=\bar{g}_{0}\left(\alpha_{s}\left(Q^{2}\right)\right)+\int_{Q^{2}}^{\bar{\Lambda}_{a}^{2}\left(Q^{2}, N\right)} \frac{d \mu^{2}}{\mu^{2}} \bar{g}\left(\alpha_{s}\left(\mu^{2}\right)\right),
$$

where $\bar{g}_{0}(\alpha)$ is an analytic function of its argument. 


\subsection{The resummed coefficient function}

Using the expression eq. (3.15) of the physical anomalous dimensions that emerges from the RG argument leads to a resummed expression for the coefficient function of the form

$$
C\left(N, Q^{2} / \mu^{2}, \alpha_{s}\left(\mu^{2}\right)\right)=C^{(c)}\left(\alpha_{s}\left(Q^{2}\right), \frac{Q^{2}}{\mu^{2}}\right) \exp \left[\int_{1}^{N^{a}} \frac{d n}{n} \int_{n \mu^{2}}^{Q^{2}} \frac{d k^{2}}{k^{2}} \bar{g}\left(\alpha_{s}\left(k^{2} / n\right)\right)\right] .
$$

Note that both $\bar{g}$ and $C^{(c)}$ are power series expansions in $\alpha_{s}$ starting at order one and zero respectively. Including the first $k$ terms in the perturbative expansion of both of them leads to resummation with $\mathrm{N}^{k} \mathrm{LL}$ accuracy.

It is customary to rewrite the resummed expression eq. (3.16) in such a way that its exponent takes the form of a Mellin transform. This is done by first, performing the change of integration variable $n=(1-x)^{-a}$, with the result

$$
C\left(N, Q^{2} / \mu^{2}, \alpha_{s}\left(\mu^{2}\right)\right)=C^{(c)}\left(\alpha_{s}\left(Q^{2}\right), \frac{Q^{2}}{\mu^{2}}\right) \exp \left[a \int_{0}^{1-\frac{1}{N}} \frac{d x}{1-x} \int_{\mu^{2}}^{Q^{2}(1-x)^{a}} \frac{d k^{2}}{k^{2}} \bar{g}\left(\alpha_{s}\left(k^{2}\right)\right)\right]
$$

and then using the identity

$$
\int_{0}^{1-\frac{1}{N}} \frac{d x}{1-x} \ln ^{p}(1-x)=-\sum_{n=0}^{p}\left(\begin{array}{l}
p \\
n
\end{array}\right) \Delta^{(n)}(1) \int_{0}^{1} d x \frac{x^{N-1}-1}{1-x} \ln ^{p-n}(1-x)+\Delta^{(p+1)}(1)+O\left(\frac{1}{N}\right)
$$

where $\Delta(z)=1 / \Gamma(z)$. One thus finds

$$
C\left(N, Q^{2} / \mu^{2}, \alpha_{s}\left(\mu^{2}\right)\right)=C^{(c)}\left(\alpha_{s}\left(Q^{2}\right), \frac{Q^{2}}{\mu^{2}}\right) \exp \left[a \int_{0}^{1} d x \frac{x^{N-1}-1}{1-x} \int_{\mu^{2}}^{Q^{2}(1-x)^{a}} \frac{d k^{2}}{k^{2}} \hat{g}\left(\alpha_{s}\left(k^{2}\right)\right)\right]
$$

where the coefficients of the expansion of the function $\hat{g}$ up to any given order are related to those of $\bar{g}$ by eq. (3.18).

It should be noted, however, that eq. (3.19) is ill-defined, because for $x$ close to 1 the $k^{2}$ integration range includes the Landau singularity of $\alpha_{s}\left(k^{2}\right)$. Hence, eq. (3.19) is only meaningful if $\hat{g}$ is expanded in powers of $\alpha_{s}\left(Q^{2}\right)$ and the $x$ integral is performed order by order up to a fixed logarithmic accuracy, i.e., in practice going back to the form eqs. (3.16)-(3.17).

The advantage of the form eq. (3.19) of the resummed expression is that the resummed exponent is then viewed as the Mellin transform of perturbative contributions that can be related to eikonal emission [20]. In the specific case of DIS, and DY (or Higgs):

$$
\begin{gathered}
C\left(N, Q^{2} / \mu^{2}, \alpha_{s}\left(\mu^{2}\right)\right)=g_{0}\left(\alpha_{s}\left(Q^{2}\right), \frac{Q^{2}}{\mu^{2}}\right) \exp \left\{n \int_{0}^{1} d x \frac{x^{N-1}-1}{1-x} \int_{\mu^{2}}^{Q^{2}(1-x)^{a}} \frac{d k^{2}}{k^{2}} A\left[\alpha_{s}\left(k^{2}\right)\right]\right. \\
\left.+\int_{0}^{1} d x \frac{x^{N-1}-1}{1-x}\left[B\left[\alpha_{s}\left(Q^{2}(1-x)^{a}\right)\right]+D\left[\alpha_{s}\left(Q^{2}(1-x)^{a}\right)\right]\right]\right\}
\end{gathered}
$$


where again the functions $A, B$ and $D$ are perturbative power series in $\alpha_{s}$. Note that this form of the resummation can be always written in the form of eq. (3.19), by expressing the $B$ and $D$ functions as the integral of a perturbative function of $\alpha_{s}$, and reabsorbing the contribution from the lower extreme of integration, which depends on $\mu^{2}$, in a redefinition of the PDF, i.e., a change of factorization scheme. Any $O\left(\alpha_{s}^{n}\right)$ contribution to $B$ and $D$ will lead to $O\left(\alpha_{s}^{n-1}\right)$ contributions to $\hat{g}$, i.e., they start to contribute at the NLL level.

In eq. (3.20) the coefficients in the expansion of $A$ are just the coefficients of the most singular contribution $\frac{1}{(1-x)}+$ to the quark or gluon splitting function expanded in powers of $\alpha_{s}$, with $n$ equal to the number of initial-state radiating partons (so $n=1$ for DIS and $n=2$ for DY and Higgs); $B$ is a universal function that collects contributions from radiation collinear to a final state parton (so it is present for DIS but not for DY or Higgs); and $D$ is a process-dependent function that (starting at $O\left(\alpha_{s}^{2}\right)$, so NNLL) includes contributions due to soft but large-angle radiation, and can be shown to vanish to all orders for DIS [21], while for DY or Higgs it can be determined by matching to a fixed-order computation.

The RG argument only determines the generic form of the dependence of the coefficient function on the kinematic variable $N$ (or $x$ ) which is resummed in the soft limit $N \rightarrow \infty$ $(x \rightarrow 1)$ : its content is that the dependence on $N$ (or $x$ ) only goes through dimensional scales $\bar{\Lambda}$ or $\Lambda$ of the form eqs. (3.5)-(3.6), and this in turn through $\alpha_{s}$ in the form of the solution to an RG equation. In the cases of DIS and DY (or Higgs) production the phase-space analysis [13] respectively leads to the identification of $\lambda^{2}=Q^{2}$ (exchanged gauge boson virtuality), $a=1$, or $Q^{2}=M^{2}$ (Higgs or gauge boson mass), $a=2$ in eqs. (3.5)-(3.6). However, this argument does not determine the form of the coefficients in the expansion of the $\bar{g}_{i}$ functions, that are fixed, at next ${ }^{k}$-to-leading logarithmic order, by comparing to a fixed next ${ }^{k}$-to-leading order result. So the form eq. (3.7) of the resummation has the advantage of emphasizing the common RG origin of all soft contributions. On the other hand, the form eq. (3.20) has the advantage of expressing the resummed result as the exponentiation of the Mellin transform of contributions that can be put in one-to-one correspondence to a fixed order calculation.

\subsection{Two-scale resummation and the transverse momentum distribution}

The multiscale case of prompt photon production considered in ref. [15], or of transverse momentum distribution discussed here, is a generalization of the approach of section 3.1, in which the process now depends on two different hard scales $Q_{1}$ and $Q_{2}$ and in the soft limit the phase space depends on two different dimensional variables of the form eq. (3.5). As discussed in section 2.1 for a transverse momentum distribution the two hard scales can be chosen as any pair out of $Q^{2}, Q p_{\mathrm{T}}, p_{\mathrm{T}}^{2}$. Because, as seen in section 2.2 the two scales eqs. (2.40)-(2.41) are respectively proportional to $Q^{2}$ and $Q p_{\mathrm{T}}$ it is natural to pick these as hard scales.

Assuming again full factorization, now also with respect to the dependence on the two different scales [12], the coefficient function takes the form

$$
\begin{aligned}
& C^{(0)}\left(N, Q_{1}^{2}, Q_{2}^{2}, \alpha_{0}, \epsilon\right) \\
& =C^{(0, c)}\left(Q_{1}^{2}, Q_{2}^{2}, \alpha_{0}, \epsilon\right) C^{\left(0, l_{1}\right)}\left(\bar{\Lambda}_{a_{1}}^{2}\left(N, \lambda_{1}^{2}\right), \alpha_{0}, \epsilon\right) C^{\left(0, l_{2}\right)}\left(\bar{\Lambda}_{a_{2}}^{2}\left(N, \lambda_{2}^{2}\right), \alpha_{0}, \epsilon\right),
\end{aligned}
$$

where the scales $\lambda_{i}$ are generally functions of the two scales $Q_{i}: \lambda_{i}=\lambda_{i}\left(N, Q_{1}^{2}, Q_{2}^{2}\right)$. 
A rerun of the same argument now leads to the physical anomalous dimension

$$
\begin{aligned}
\gamma\left(N, 1, Q_{2}^{2} / Q_{1}^{2}, \alpha_{s}\left(Q_{2}^{2}\right), \epsilon\right)= & \bar{g}_{0}\left(\alpha\left(Q_{1}^{2}\right), Q_{2}^{2}\right) \\
& +\int_{Q_{1}^{2}}^{\bar{\Lambda}_{a_{1}}^{2}\left(N, \lambda_{1}^{2}\right)} \frac{d \mu^{2}}{\mu^{2}} \bar{g}_{1}\left(\alpha_{s}\left(\mu^{2}\right)\right)+\int_{Q_{1}^{2}}^{\bar{\Lambda}_{a_{2}}^{2}\left(N, \lambda_{2}^{2}\right)} \frac{d \mu^{2}}{\mu^{2}} \bar{g}_{2}\left(\alpha_{s}\left(\mu^{2}\right)\right) .
\end{aligned}
$$

Note that when solving the renormalization-group equation we have chosen one of the two hard scales, namely $Q_{1}$, both as renormalization and factorization scale; of course nothing prevents one from re-expressing the result for generic choices of these scales. We thus end up with a resummed coefficient function of the form

$$
\begin{aligned}
& C\left(N, Q_{1}^{2} / \mu^{2}, Q_{2}^{2} / \mu^{2}, \alpha_{s}\left(\mu^{2}\right)\right)=C^{(c)}\left(\alpha_{s}\left(Q^{2}\right), \frac{Q^{2}}{\mu^{2}}\right) \\
& \quad \times \exp \left[\int_{1}^{N_{1}^{a}} \frac{d n_{1}}{n_{1}} \int_{n_{1} k^{2}}^{\lambda_{1}^{2}} \frac{d k_{1}^{2}}{k_{1}^{2}} \bar{g}_{1}\left(\alpha_{s}\left(k_{1}^{2}\right)\right)+\int_{1}^{N_{2}^{a}} \frac{d n_{2}}{n_{2}} \int_{n_{2} k^{2}}^{\lambda_{2}^{2}} \frac{d k_{2}^{2}}{k_{2}^{2}} \bar{g}_{2}\left(\alpha_{s}\left(k^{2}\right)\right)\right],
\end{aligned}
$$

where the functions $\bar{g}_{i}$ are power series in $\alpha_{s}$ of the form of eq. (3.14).

We come now to the case of the DY or Higgs transverse momentum distributions, discussed in the previous section. In this case, the leading-order process always has a parton in the final state, and we must thus distinguish between two partonic subchannels, according to the species (quark or gluon) of final-state parton. The final-state parton is necessarily of the same species of one of the initial-state partons. The other initial-state parton is a quark for the DY process, and a gluon for Higgs. The argument of section 2.2 then shows that the phase space factors into a phase space with DY-like kinematics, characterized by the scale $\Lambda_{\mathrm{DY}}^{2}$ eq. (2.40), and a phase space with DIS-like kinematics, characterized by the scale $\Lambda_{\text {DIS }}^{2}$ eq. (2.41). Assuming factorization of the full amplitude in the soft limit the coefficient function factorizes according to eq. (3.21). We thus arrive at the resummed expression

$$
\begin{aligned}
& C_{i j}\left(N, Q^{2} / \mu^{2}, Q p_{\mathrm{T}} / \mu^{2}, \alpha_{s}\left(\mu^{2}\right)\right)=C^{(c)}\left(\alpha_{s}\left(Q^{2}\right), \frac{Q^{2}}{\mu^{2}}\right) \\
& \quad \times \exp \left[\int_{1}^{N^{2}} \frac{d n_{1}}{n_{1}} \int_{n_{1} \mu^{2}}^{Q^{2}} \frac{d k_{1}^{2}}{k_{1}^{2}} \bar{g}_{1}^{(i)}\left(\alpha_{s}\left(k_{1}^{2}\right)\right)+\int_{1}^{N} \frac{d n_{2}}{n_{2}} \int_{n_{2} \mu^{2}}^{Q p_{\mathrm{T}}} \frac{d k_{2}^{2}}{k_{2}^{2}} \bar{g}_{2}^{(j)}\left(\alpha_{s}\left(k_{2}^{2}\right)\right)\right],
\end{aligned}
$$

where $Q^{2}$ is given by eq. (2.4), and $j$ denotes the species of outgoing parton in the Born process, and $i$ is a quark for DY and a gluon for Higgs.

Using further the expression eq. (3.20) of the resummed DY and DIS coefficient functions we get

$$
\begin{aligned}
& C_{i j}\left(N, Q^{2} / \mu^{2}, Q p_{\mathrm{T}} / \mu^{2}, \alpha_{s}\left(\mu^{2}\right)\right)=g_{0}^{i j}\left(\alpha_{s}\left(Q^{2}\right), \frac{Q^{2}}{\mu^{2}}\right) \\
& \quad \times \exp \left\{\int _ { 0 } ^ { 1 } d x \frac { x ^ { N - 1 } - 1 } { 1 - x } \left[D^{i}\left[\alpha_{s}\left(Q^{2}(1-x)^{2}\right)\right]+\int_{\mu^{2}}^{Q^{2}(1-x)^{2}} \frac{d k^{2}}{k^{2}} A^{i}\left[\alpha_{s}\left(k^{2}\right]\right]\right.\right. \\
& \left.\quad+\int_{0}^{1} d x \frac{x^{N-1}-1}{1-x}\left[B^{j}\left[Q p_{\mathrm{T}}(1-x)\right]+\int_{\mu^{2}}^{Q p_{\mathrm{T}}(1-x)} \frac{d k^{2}}{k^{2}} A^{j}\left[\alpha_{s}\left(k^{2}\right)\right]\right]\right\}
\end{aligned}
$$


Note that the function $B$ that characterizes radiation from the outgoing parton now also carries an index according to whether this is a quark or gluon: so it corresponds for instance to either standard DIS (quark) or deep-inelastic Higgs production in photon-gluon fusion (gluon) [20].

Equations (3.24)-(3.25) are the main result of this paper and are the desired all-order generalization of the NLL resummation of ref. [8]. In the next section we will compare them directly to the known NLO result and verify that they agree with it, which may not be immediately obvious.

\section{Resummed results}

Explicit resummed expressions can be obtained by performing the integrals in eqs. (3.25)(3.24), in terms of the coefficients of the expansion of the QCD $\beta$ function and of the functions $A_{i}, B_{i}, D_{i}$ (eq. (3.25)) or $g_{i}$ (eq. (3.24)) in powers of $\alpha_{s}$. These expressions were given up to NLL in ref. [8], and are given at NNLL in the appendix. Below we check that our results agree with previous known resummed or fixed-order results.

\subsection{Next-to-leading resummation}

As repeatedly mentioned, threshold resummation of transverse momentum distributions up to NLL accuracy was given in ref. [8]. In that reference, the resummed expression of the coefficient function is written in the form

$$
C_{i j}\left(N, Q^{2} / \mu^{2}, Q p_{\mathrm{T}} / \mu^{2}, \alpha_{s}\left(\mu^{2}\right)\right)=g_{0}^{i j}\left(\alpha_{s}\left(Q^{2}\right)\right) \Delta_{i}\left(Q p_{\mathrm{T}}\right) \Delta_{j}\left(Q p_{\mathrm{T}}\right) J_{k}\left(Q p_{\mathrm{T}}\right) \Delta_{i j k}^{\mathrm{int}}\left(Q p_{\mathrm{T}}\right)
$$

where $k$ denotes the Born-level outgoing parton and

$$
\begin{aligned}
\ln \Delta_{i}\left(Q p_{\mathrm{T}}\right) & =\int_{0}^{1} d x \frac{x^{N-1}-1}{1-x} \int_{\mu^{2}}^{Q p_{\mathrm{T}}(1-x)^{2}} \frac{d q^{2}}{q^{2}} A^{i}\left(\alpha_{s}\left(q^{2}\right)\right) \\
\ln J_{k}\left(Q p_{\mathrm{T}}\right) & =\int_{0}^{1} d x \frac{x^{N-1}-1}{1-x}\left[\int_{Q p_{\mathrm{T}}(1-x)^{2}}^{Q p_{\mathrm{T}}(1-x)} \frac{d q^{2}}{q^{2}} A^{k}\left(\alpha_{s}\left(q^{2}\right)\right)+B^{k}\left(\alpha_{s}\left(Q p_{\mathrm{T}}(1-x)\right)\right)\right] \\
\ln \Delta_{\text {int }}^{i j k}\left(Q p_{\mathrm{T}}\right) & =\int_{0}^{1} d x \frac{x^{N-1}-1}{1-x} D^{i j k}\left(\alpha_{s}\left(Q p_{\mathrm{T}}(1-x)^{2}\right)\right)
\end{aligned}
$$

where the functions $A^{i}$ and $B^{i}$ are as in eq. (3.25) and

$$
D_{1}^{i j k}=-\left(A_{1}^{i}+A_{1}^{j}-A_{1}^{k}\right) \ln \frac{p_{\mathrm{T}}}{Q},
$$

with $A_{1}^{i}$ the first-order coefficient in the expansion of the function $A^{i}\left(\alpha_{s}\right)$ in powers of its argument. Also as mentioned, what is called $Q^{2}$ in ref. [8] corresponds to what we call $Q p_{\mathrm{T}}$ here.

In order to compare with our resummed expression eq. (3.25), note that

$$
\alpha_{s}\left(Q p_{\mathrm{T}}(1-x)^{2}\right) \ln \frac{p_{\mathrm{T}}}{Q}=\int_{Q^{2}(1-x)^{2}}^{Q p_{\mathrm{T}}(1-x)^{2}} \frac{d q^{2}}{q^{2}} \alpha_{s}\left(q^{2}\right)+O\left(\alpha_{s}^{2}\left(Q^{2}\right)\right) .
$$


It follows that, to NLL accuracy, we can reabsorb the $\Delta_{\text {int }}^{i j k}\left(Q p_{\mathrm{T}}\right)$ term into a change of scale of the $\Delta_{i}\left(Q p_{\mathrm{T}}\right)$ and $J_{k}\left(Q p_{\mathrm{T}}\right)$ functions. Namely, eq. (4.1) can be rewritten in the rather simpler form

$$
C_{i j}\left(N, Q^{2} / \mu^{2}, Q p_{\mathrm{T}} / \mu^{2}, \alpha_{s}\left(\mu^{2}\right)\right)=g_{0}^{i j}\left(\alpha_{s}\left(Q^{2}\right)\right) \Delta_{i}\left(Q^{2}\right) \Delta_{j}\left(Q^{2}\right) \bar{J}_{k}\left(Q^{2}, Q p_{\mathrm{T}}\right),
$$

where

$$
\ln \bar{J}_{k}\left(Q^{2}, Q p_{\mathrm{T}}\right)=\int_{0}^{1} d x \frac{x^{N-1}-1}{1-x}\left[\int_{Q^{2}(1-x)^{2}}^{Q p_{\mathrm{T}}(1-x)} \frac{d q^{2}}{q^{2}} A^{k}\left(\alpha_{s}\left(q^{2}\right)\right)+B^{k}\left[\alpha_{s}\left(Q p_{\mathrm{T}}(1-x)\right)\right]\right] .
$$

Exploiting the fact that, as mentioned, the outgoing parton $k$ is always equal to either $i$ or to $j$, and using the same convention as in eqs. (3.23)-(3.25), i.e. assuming that the outgoing parton is $j$ we then get

$$
\begin{aligned}
& \ln \Delta_{j}\left(Q^{2}\right)+\ln \bar{J}_{j}\left(Q^{2}, Q p_{\mathrm{T}}\right) \\
& =\int_{0}^{1} d x \frac{x^{N-1}-1}{1-x}\left[\int_{\mu^{2}}^{Q p_{\mathrm{T}}(1-x)} \frac{d q^{2}}{q^{2}} A^{j}\left(\alpha_{s}\left(q^{2}\right)\right)+B^{j}\left[\alpha_{s}\left(Q p_{\mathrm{T}}(1-x)\right)\right]\right] .
\end{aligned}
$$

Recalling that the function $D$ in eq. (3.25) starts at $O\left(\alpha_{s}^{2}\right)$, at the NLL level eq. (4.7) is seen to coincide with eq. (3.25), with $\Delta_{i}\left(Q^{2}\right)$ identified with the Drell-Yan like term, and $\ln \Delta_{j}\left(Q^{2}\right)+\ln \bar{J}_{j}\left(Q^{2}, Q p_{\mathrm{T}}\right)$ eq. (4.9) identified with the DIS-like term.

It is interesting to observe that the renormalization-group argument makes clear that to all orders the dependence on $p_{\mathrm{T}}$ is only through the argument of $\alpha_{s}$ evaluated at the scale $\Lambda_{\text {DIS }}^{2}$ eq. (2.41). Note however that it is always possible even at the NNLL level and beyond to rewrite the resummed expression eq. (3.25) in the form of ref. [8], eq. (4.1). Indeed, this simply corresponds to choosing $Q p_{\mathrm{T}}(1-x)^{2}$ instead of $Q^{2}(1-x)^{2}$ as upper limit of integration in eq. (3.25). This then produces and extra contribution of the form of the right-hand side of eq. (4.6), but now in general with higher order powers of $\alpha_{s}\left(q^{2}\right)$ under the integral. The integral can be performed and expressed in terms of $\ln \frac{p_{\mathrm{T}}}{Q}$ and $\alpha_{s}\left(Q p_{\mathrm{T}}(1-x)^{2}\right)$, thereby leading to higher order contributions to $\ln \Delta_{\text {int }}^{i j k}\left(Q p_{\mathrm{T}}\right)$.

\subsection{Comparison to fixed-order calculation}

As discussed in the introduction, in ref. [8] the expansion of the resummed result up to NLO was compared numerically to the fixed order result $O\left(\alpha_{s}^{2}\right)$ for Higgs production in gluon fusion of ref. [16]. At $O\left(\alpha_{s}^{2}\right)$, because the LO is $O\left(\alpha_{s}\right)$, the coefficient function eq. (3.20) must be expanded up to $O\left(\alpha_{s}\right)$, and it then contains a LL $\ln ^{2} N$ term, and, at NLL, $\ln N$ and constant contributions. The constant is fixed by matching to the fixed-order calculation, and it determines the contribution to $g_{0}$ in eq. (3.20) that is linear in $\alpha_{s}$.

In a pure RG approach, in which only the functional form of the resummation eq. (3.24) or eq. (3.25) is known, the coefficients of the NLL logarithmic terms are also fixed by comparison to the fixed NLO, and then used to predict results at NNLO and beyond. However, if one instead uses the form eq. (3.20) of the resummed result, all the logarithmic coefficients up to NLL accuracy (so all logarithmic coefficients at NLO) are predicted in terms of the universal, process-independent functions $A$ and $B$. 
We thus get a nontrivial check by comparing the coefficients of the logarithmically enhanced terms of the fixed-order result to those obtained by using the form eq. (3.25) of the resummation, truncated to NLL, and expanded to first order in $\alpha_{s}$. We perform this check in the gluon-gluon channel, where we have

$$
\begin{gathered}
C_{g g}\left(N, Q^{2} / \mu^{2}, Q p_{\mathrm{T}} / \mu^{2}, \alpha_{s}\left(\mu^{2}\right)\right)=1+\frac{\alpha_{s}}{2 \pi}\left[\int_{0}^{1} d x \frac{x^{N-1}-1}{1-x} \int_{\mu^{2}}^{Q^{2}(1-x)^{2}} \frac{d k^{2}}{k^{2}} A_{1}^{g}\right. \\
\left.+\int_{0}^{1} d x \frac{x^{N-1}-1}{1-x}\left(B_{1}^{g}+\int_{\mu^{2}}^{Q p_{\mathrm{T}}(1-x)} \frac{d k^{2}}{k^{2}} A_{1}^{g}\right)\right]+O\left(\frac{\alpha_{s}}{2 \pi}\right)^{2}+O\left(\ln ^{0} N\right)
\end{gathered}
$$

having noticed that the $D$ function starts at $O\left(\alpha_{s}^{2}\right)$, that the contribution proportional to $A_{2}$, even though is the same order as $B_{1}$, also starts contributing at $O\left(\alpha_{s}^{2}\right)$, and finally that the prefactor function $g_{0}$ only starts contributing to logarithmically enhanced terms at order $\alpha_{s}^{2}$.

The $k^{2}$ integrals are trivial to perform, and the $x$ integrals can be obtained by differentiating the generating function

$$
J_{\varepsilon}=\int_{0}^{1} d x \frac{x^{k-1}}{(1-x)^{1-\epsilon}}=\frac{\Gamma(k) \Gamma(\epsilon)}{\Gamma(k+\epsilon)}
$$

see e.g. ref. [13]. We then get

$$
C_{g g}\left(N, Q^{2} / \mu^{2}, Q p_{\mathrm{T}} / \mu^{2}, \alpha_{s}\left(\mu^{2}\right)\right)=1+\frac{\alpha_{s}}{2 \pi}\left[c_{2} \ln ^{2} N+c_{1}\left(p_{\mathrm{T}}^{2}\right) \ln N+O\left(\ln ^{0} N\right)\right]+O\left(\alpha_{s}^{2}\right)
$$

with

$$
\begin{aligned}
c_{2} & =3 A_{1}^{g} \\
c_{1}\left(p_{\mathrm{T}}^{2}\right) & =2 A_{1}^{g}\left(3 \gamma+2 \ln \frac{\mu^{2}}{Q^{2}}-\ln \frac{p_{\mathrm{T}}}{Q}\right)-2 B_{1}^{g} .
\end{aligned}
$$

Extracting the corresponding coefficients from the fixed-order NLO result of ref. [16] is not entirely trivial, because the result given there is fully differential, so it must be integrated in rapidity in order to get the transverse momentum distribution. The computation is presented in appendix B. The final result is given in eqs. (B.10)-(B.11), and it matches the expansion of the resummed result eqs. (4.13)-(4.14).

\section{Outlook}

In this paper we have derived threshold resummation for transverse momentum distributions by using a renormalization group approach to resummation originally developed in refs. $[13,14]$, extended to prompt photon production in ref. [15], and here to the more general case of processes with two soft scales, of which prompt photon production and transverse momentum distributions are specific instances. From a technical point of view, the main difference to previous work is that a transverse momentum distribution not only has two different soft scales in the threshold limit, but also two distinct hard scales, which 
makes the phase-space analysis that is at the heart of the renormalization group argument somewhat more involved.

Our result generalizes to all orders previous NLL results of ref. [8]. In comparison to them, the renormalization group approach leads to a somewhat more compact resummed expression, also at NLL. In paricular, there is no need to introduce a process-dependent interference contribution specific of transverse momentum distribution [8]: this is reabsorbed by a suitable choice of scale. Rather, the result is entirely expressed in terms of the resummation for the Drell-Yan-like and DIS-like structures in which it is decomposed.

The resummed results presented here may be used, matched to fixed order calculations, in order to accelerate their perturbative convergence, and also, for the construction of approximations to unknown higher fixed-order results [22]. On a more theoretical note, these results can be useful in the construction of joint resummed results, in which soft resummation is combined with high-energy resummation [23] or with transverse-momentum resummation [5]. Specifically, they could shed light on the common origin of soft and transverse-momentum resummed logarithms, thereby leading to more powerful joint resummed expression. This is the subject of ongoing investigations and it will be left to future work.

\section{Acknowledgments}

SF thanks Kirill Kudashkin and Tanjona Rabemananjara for several discussions. SF is supported by the European Research Council under the European Union's Horizon 2020 research and innovation Programme (grant agreement n.740006). The work of GR is supported by the Italian Ministry of Research (MIUR) under grant PRIN 20172LNEEZ.

\section{A Explicit resummed results}

The exponent of a resummed coefficient function is conveniently written as a power expansion in $\alpha_{s}\left(Q^{2}\right)$ at fixed $\alpha_{s}\left(Q^{2}\right) \ln N$. To this purpose, we define a resummation variable

$$
\lambda=b_{0} \alpha_{s}\left(Q^{2}\right) \ln N
$$

where $b_{0}$ is the first coefficient in the expansion of the QCD $\beta$ function:

$$
\begin{aligned}
\mu^{2} \frac{d \alpha_{s}\left(\mu^{2}\right)}{d \mu^{2}} & =-b_{0} \alpha_{s}^{2}\left(\mu^{2}\right)-b_{1} \alpha_{s}^{3}\left(\mu^{2}\right)-b_{2} \alpha_{s}^{4}\left(\mu^{2}\right)+O\left(\alpha_{s}^{5}\right) \\
b_{0} & =\frac{11 C_{A}-4 T_{f} n_{f}}{12 \pi} \\
b_{1} & =\frac{\left.17 C_{A}^{2}-10 C_{A} n_{f} T_{f}-6 C_{F} n_{f} T_{f}\right)}{24 \pi^{2}} \\
b_{2} & =\frac{1}{128 \pi^{3}}\left(2857-\frac{5033}{9} n_{f}+\frac{325}{27} n_{f}^{2}\right)
\end{aligned}
$$

The resummed exponent, i.e. the logarithm of the resummed coefficient function, is then written as an expansion in powers of $\alpha_{s}$ at fixed $\lambda$, so that including the first $k+1$ 
orders of this expansion, that we call $g_{k}^{i j}\left(\lambda, \frac{p_{T}}{Q}\right)$, resummation at $\mathrm{N}^{k} \mathrm{LL}$ level is obtained. Up to and including NNLL we thus get

$$
\begin{aligned}
& C_{i j}\left(N, Q^{2} / \mu^{2}, Q p_{\mathrm{T}} / \mu^{2}, \alpha_{s}\left(\mu^{2}\right)\right)=g_{0}^{i j}\left(\alpha_{s}\left(Q^{2}\right)\right) \\
& \quad \exp \left[\frac{1}{\alpha_{s}\left(Q^{2}\right)} g_{1}^{i j}\left(\lambda, \frac{p_{\mathrm{T}}}{Q}\right)+g_{2}^{i j}\left(\lambda, \frac{p_{\mathrm{T}}}{Q}\right)+\alpha_{s}\left(Q^{2}\right) g_{3}^{i j}\left(\lambda, \frac{p_{\mathrm{T}}}{Q}\right)+O\left(\alpha_{s}^{3}\right)\right] .
\end{aligned}
$$

Note that the expansion of $g_{1}$ in powers of $\alpha_{s}$ starts at order $\alpha_{s}^{2}$ while the expansion of all $g_{i}$ with $i>1$ starts at $O\left(\alpha_{s}\right)$. In order to achieve $\mathrm{N}^{k} \mathrm{LL}$ accuracy of the coefficient function, it is necessary also to include the first $k+1$ orders in the expansion of the prefactor $g_{0}^{i j}\left(\alpha_{s}\left(Q^{2}\right)\right)$ in powers of $\alpha_{s}$. At $\mathrm{N}^{k} \mathrm{LL}$ order the coefficients of all terms proportional to $\alpha_{s}^{n} \ln ^{2 n-2 k} N$ are then correctly predicted.

The coefficients $g_{k}^{i j}\left(\lambda, \frac{p_{\mathrm{T}}}{Q}\right)$ are given in terms of the functions $A^{i}\left(\alpha_{s}\right), B^{i}\left(\alpha_{s}\right), D^{i}\left(\alpha_{s}\right)$ defined in eq. (3.20), which in turn have perturbative expansions

$$
\begin{aligned}
& A^{i}\left(\alpha_{s}\right)=\sum_{n=1}^{\infty} A_{n}^{i}\left(\frac{\alpha_{s}}{\pi}\right)^{n} \\
& B^{i}\left(\alpha_{s}\right)=\sum_{n=1}^{\infty} B_{n}^{i}\left(\frac{\alpha_{s}}{\pi}\right)^{n} \\
& D^{i}\left(\alpha_{s}\right)=\sum_{n=2}^{\infty} D_{n}^{i}\left(\frac{\alpha_{s}}{\pi}\right)^{n} .
\end{aligned}
$$

The universal coefficients $A^{i}$ and $B^{i}$ and the coefficients $D^{i}$ for Drell-Yan and Higgs production are given up to $\mathrm{N}^{3} \mathrm{LL}$ in ref. [20].

Substituting the expansions eqs. (A.7)-(A.9) in the resummed expression eq. (3.20) we get, up to NNLL,

$$
\begin{aligned}
g_{1}^{i j}\left(\lambda, \frac{p_{\mathrm{T}}}{Q}\right)= & \frac{1}{2 b_{0}^{2} \pi}\left[A_{1}^{i}(2 \lambda+(1-2 \lambda) \ln (1-2 \lambda))+2 A_{1}^{j}(\lambda+(1-\lambda) \ln (1-\lambda))\right] \quad \text { (A.10) } \\
g_{2}^{i j}\left(\lambda, \frac{p_{\mathrm{T}}}{Q}\right)= & \frac{A_{1}^{i}}{4 b_{0}^{3} \pi}\left[4 \lambda\left(b_{1}+b_{0}^{2} \ln \frac{\mu^{2}}{Q^{2}}\right)+\ln (1-2 \lambda)\left(2 b_{1}-4 b_{0}^{2} \gamma+b_{1} \ln (1-2 \lambda)\right)\right] \\
& -\frac{A_{2}^{i}}{2 b_{0}^{2} \pi^{2}}[2 \lambda+\ln (1-2 \lambda)] \\
& +\frac{A_{1}^{j}}{2 b_{0}^{3} \pi}\left[2 \lambda\left(b_{1}+b_{0}^{2} \ln \frac{\mu^{2}}{Q^{2}}\right)+2\left(b_{1}+b_{0}^{2}\left(\ln \frac{p_{\mathrm{T}}}{Q}-\gamma\right)\right) \ln (1-\lambda)+b_{1} \ln ^{2}(1-\lambda)\right] \\
& -\frac{A_{2}^{j}}{b_{0}^{2} \pi^{2}}[\lambda+\ln (1-\lambda)]+\frac{B_{1}^{j}}{b_{0} \pi} \ln (1-\lambda) .
\end{aligned}
$$




$$
\begin{aligned}
& g_{3}^{i j}\left(\lambda, \frac{p_{\mathrm{T}}}{Q}\right)=\frac{1}{12 b_{0}^{4} \pi^{3}}\left\{\frac { 1 } { 2 \lambda - 1 } \left[6 b _ { 0 } \left(2 b_{0} \lambda\left(-\lambda A_{3}^{i}+b_{0} \pi D_{2}^{i}\right)\right.\right.\right. \\
& \left.+\pi A_{2}^{i}\left(2 b_{1} \lambda(1+\lambda)-2 b_{0}^{2} \lambda\left(2 \gamma+\ln \frac{\mu^{2}}{Q^{2}}-2 \lambda \ln \frac{\mu^{2}}{Q^{2}}\right)+b_{1} \ln (1-2 \lambda)\right)\right) \\
& \quad+\pi^{2} A_{1}^{i}\left(-2 \lambda\left(-12 b_{0}^{2} b_{1} \gamma-6 b_{0} b_{2}(\lambda-1)+6 b_{1}^{2} \lambda+b_{0}^{4}\left(12 \gamma^{2}+(-3+6 \lambda) \ln ^{2} \frac{\mu^{2}}{Q^{2}}+2 \pi^{2}\right)\right)\right. \\
& \left.\left.\quad-3 \ln (1-2 \lambda)\left(2 b_{0} b_{2}-4 b_{0}^{2} b_{1} \gamma+4 b_{1}^{2} \lambda-4 b_{0} b_{2} \lambda+b_{1}^{2} \ln (1-2 \lambda)\right)\right)\right] \\
& +\frac{1}{\lambda-1}\left[6 b _ { 0 } \left(\lambda\left(b_{1}(2+\lambda)-2 b_{0}^{2}\left(\gamma-\ln \frac{p_{\mathrm{T}}}{Q}+(1-\lambda) \ln \frac{\mu^{2}}{Q^{2}}\right)\right) \pi A_{2}^{j}\right.\right. \\
& +b_{0} \lambda\left(-\lambda A_{3}^{j}+2 \pi\left(-\left(b_{1}-b_{0}^{2}\left(\gamma-\ln \frac{p_{\mathrm{T}}}{Q}\right)\right) \pi B_{1}^{j}+b_{0} B_{2}^{j}\right)\right) \\
& \left.+2 b_{1} \pi\left(A_{2}^{j}-b_{0} \pi B_{1}^{j}\right) \ln (1-\lambda)\right) \\
& +\pi^{2} A_{1}^{j}\left(\lambda \left(6 b_{0} b_{2}(\lambda-2)-6 b_{1}^{2} \lambda+12 b_{0}^{2} b_{1}\left(\gamma-\ln \frac{p_{\mathrm{T}}}{Q}\right)\right.\right. \\
& \left.\quad-b_{0}^{4}\left(6\left((\lambda-1) \ln ^{2} \frac{\mu^{2}}{Q^{2}}+\left(\gamma-\ln \frac{p_{\mathrm{T}}}{Q}\right)^{2}\right)+\pi^{2}\right)\right)-6 \ln (1-\lambda)\left(2 \left(b_{1}^{2} \lambda+b_{0}\left(b_{2}-b_{2} \lambda\right)\right.\right. \\
& \left.\left.\left.\left.\left.-b_{0}^{2} b_{1}\left(\gamma-\ln \frac{p_{\mathrm{T}}}{Q}\right)\right)+b_{1}^{2} \ln (1-\lambda)\right)\right)\right\}\right\}
\end{aligned}
$$

In these expressions, $\mu^{2}$ is the factorization scale, while the renormalization scale $\mu_{\mathrm{R}}^{2}$ was taken to be equal to $Q^{2}$. The dependence on the renormalization scale can easily be restored by shifting the argument of $\alpha_{s}$. To NNLL accuracy, this amounts to replacing

$$
\alpha_{s}\left(Q^{2}\right)=\alpha_{s}\left(\mu_{\mathrm{R}}^{2}\right)+\alpha_{s}^{2}\left(\mu_{\mathrm{R}}^{2}\right) b_{0} \ln \frac{\mu_{\mathrm{R}}^{2}}{Q^{2}}+\alpha_{s}^{3}\left(\mu_{\mathrm{R}}^{2}\right)\left(b_{1} \ln \frac{\mu_{\mathrm{R}}^{2}}{Q^{2}}+b_{0}^{2} \ln ^{2} \frac{\mu_{\mathrm{R}}^{2}}{Q^{2}}\right)
$$

in eq. (A.6), and expanding in powers of $\alpha_{s}\left(\mu_{\mathrm{R}}^{2}\right)$ at

$$
\lambda_{\mathrm{R}}=b_{0} \alpha_{s}\left(\mu_{\mathrm{R}}^{2}\right) \ln N
$$

fixed. As a consequence, the functions $g_{2}, g_{3}$ are modified as follows:

$$
g_{2}^{i j}\left(\lambda, \frac{p_{\mathrm{T}}}{Q}\right) \rightarrow g_{2}^{i j}\left(\lambda_{\mathrm{R}}, \frac{p_{\mathrm{T}}}{Q}\right)-\frac{1}{2 \pi b_{0}}\left[A_{1}^{i}\left(2 \lambda_{\mathrm{R}}+\ln \left(1-2 \lambda_{\mathrm{R}}\right)\right)+2 A_{1}^{j}\left(\lambda_{\mathrm{R}}+\ln \left(1-\lambda_{\mathrm{R}}\right)\right)\right] \ln \frac{\mu_{\mathrm{R}}^{2}}{Q^{2}}
$$




$$
\begin{aligned}
g_{3}^{i j}\left(\lambda, \frac{p_{\mathrm{T}}}{Q}\right) \rightarrow & g_{3}^{i j}\left(\lambda_{\mathrm{R}}, \frac{p_{\mathrm{T}}}{Q}\right)+\frac{\lambda_{\mathrm{R}}^{2}\left(2\left(1-\lambda_{\mathrm{R}}\right) A_{1}^{i}+\left(1-2 \lambda_{\mathrm{R}}\right) A_{1}^{j}\right)}{2 \pi\left(1-\lambda_{\mathrm{R}}\right)\left(1-2 \lambda_{\mathrm{R}}\right)} \ln ^{2} \frac{\mu_{\mathrm{R}}^{2}}{Q^{2}} \\
& +\frac{1}{2 \pi^{2} b_{0}^{2}\left(1-\lambda_{\mathrm{R}}\right)\left(1-2 \lambda_{\mathrm{R}}\right)}[ \\
& -\pi A_{1}^{i}\left(1-\lambda_{\mathrm{R}}\right)\left(2 \lambda_{\mathrm{R}}\left(b_{1}-b_{0}^{2}\left(2 \gamma+\ln \frac{\mu^{2}}{Q^{2}}\left(1-2 \lambda_{\mathrm{R}}\right)\right)\right)+b_{1} \ln \left(1-2 \lambda_{\mathrm{R}}\right)\right) \\
& +2 b_{0} \lambda_{\mathrm{R}}\left(2\left(1-\lambda_{\mathrm{R}}\right) \lambda_{\mathrm{R}} A_{2}^{i}+\left(1-2 \lambda_{\mathrm{R}}\right)\left(\lambda_{\mathrm{R}} A_{2}^{j}-b_{0} \pi B_{1}^{j}\right)\right) \\
& \left.-2 \pi A_{1}^{j}\left(1-2 \lambda_{\mathrm{R}}\right)\left(\lambda_{\mathrm{R}}\left(b_{1}-b_{0}^{2}\left(\gamma+\left(1-\lambda_{\mathrm{R}}\right) \ln \frac{\mu^{2}}{Q^{2}}-\ln \frac{p_{\mathrm{T}}}{Q}\right)\right)+b_{1} \ln \left(1-\lambda_{\mathrm{R}}\right)\right)\right] \\
& \times \ln \frac{\mu_{\mathrm{R}}^{2}}{Q^{2}}
\end{aligned}
$$

Results up to NLO were already given in ref. [8]. We have shown in section 4.1 that our results are in agreement with that reference, and we further checked explicitly that eqs. (A.10), (A.11), (A.15) agree with results presented there, once account is taken for the different notation and choice of hard scale. The NNLL result eqs. (A.12), (A.16) is a new result of this paper.

\section{B The NLO transverse momentum distribution}

The NLO computation of ref. [16] determines the double differential cross section in transverse momentum and rapidity at the partonic level for Higgs production in gluon fusion. The computation is performed in the heavy top mass limit; however, in the soft limit the heavy top approximation is exact. In order to compare this result to the resummed result we must integrate in rapidity the double differential cross section.

We concentrate on the gluon-gluon channel, for which the expansion of the resummed result was given in section 4.2. In ref. [16] the double differential partonic cross-section is written as

$$
\frac{d \hat{\sigma}_{i j}}{d p_{\mathrm{T}}^{2} d y}=\frac{\sigma_{0}}{\hat{s}}\left[\frac{\alpha_{s}\left(\mu_{\mathrm{R}}^{2}\right)}{2 \pi} G_{g g}^{(1)}+\left(\frac{\alpha_{s}\left(\mu_{\mathrm{R}}^{2}\right)}{2 \pi}\right)^{2} G_{g g}^{(2)}+O\left(\left(\alpha_{s}\right)^{3}\right)\right],
$$

where $\sigma_{0}$ is the leading-order total cross-section, and the logarithmically enhanced (either in the soft or the small $p_{\mathrm{T}}$ limit) contribution to the NLO term $G_{g g}^{(2)}$ is given in eq. (3.17) of ref. [16].

The rapidity integral can be computed by performing a change of variable suggested in ref. [24] from rapidity $y$ to $K^{2}$, the invariant mass of the two final state parton system. The two variables are related by

$$
\sinh y= \pm \frac{\sqrt{\left(\hat{s}+m^{2}-K^{2}\right)^{2}-4 \hat{s} E_{\mathrm{T}}^{2}}}{2 \sqrt{\hat{s}} E_{\mathrm{T}}}
$$

By a further change of variables $K^{2} \rightarrow q=K^{2} / K_{\max }^{2}$, where $K_{\max }^{2}$ is the upper bound of the $K^{2}$ integral, the rapidity integrals, up to terms of order $O(1-x)$, are all reduced to 
integrals of the general form

$$
I_{k}=\int_{0}^{1}\left[\frac{\ln ^{k} q}{q}\right]_{+} \frac{1}{\sqrt{(1-q)}} d q .
$$

This is done by starting with the plus distributions contained in $G_{g g}^{(2)}$ of ref. [16], which are expressed in terms of the scaling variables $z_{t}$ and $z_{u}$ respectively given by

$$
z_{t}=\frac{-t}{K^{2}-t} ; \quad z_{u}=\frac{-u}{K^{2}-u},
$$

and exploiting the identities between plus distributions

$$
\begin{aligned}
\frac{z_{t}}{-t}\left(\frac{1}{1-z_{t}}\right)_{+} & =\frac{1}{K_{\max }^{2}}\left\{\left[\frac{1}{q}\right]_{+}+\delta(q) \ln \frac{K_{\max }^{2}}{-t}\right\} \\
\frac{z_{t}}{-t}\left(\frac{\ln \left(1-z_{t}\right)}{1-z_{t}}\right)_{+} & =\frac{1}{K_{\max }^{2}}\left\{\left[\frac{\ln (q)}{q}\right]_{+}+\ln \frac{K_{\max }^{2} z_{t}}{-t}\left[\frac{1}{q}\right]_{+}+\frac{\delta(q)}{2} \ln ^{2} \frac{K_{\max }^{2}}{-t}\right\}
\end{aligned}
$$

and similarly for $z_{u}$.

The integrals $I_{k}$ eq. (B.3) can be computed by differentiating with respect to $\epsilon$ the generating function

$$
I_{\epsilon}=\int_{0}^{1} \frac{1}{q^{-1+\epsilon}}\left[\frac{1}{\sqrt{(1-q)}}-1\right]=\frac{\Gamma\left(\frac{1}{2}\right) \Gamma(\epsilon)}{\Gamma\left(\frac{1}{2}+\epsilon\right)}-\frac{1}{\epsilon} .
$$

This leads to an expression of the differential cross section that contains threshold logarithms of the form [5] $\left(\frac{\ln ^{k}(1-x)}{\sqrt{(1-x)(1-a x)}}\right)$, where $a=\left(\frac{E_{\mathrm{T}}+p_{\mathrm{T}}}{m}\right)^{2}$, whose Mellin transform can be computed from the generating function

$$
D_{\eta}^{a}(N)=\int_{0}^{1} \frac{x^{N}}{\sqrt{(1-x)(1-a x)}}(1-x)^{\eta} d x=\frac{\Gamma(N) \Gamma\left(\eta+\frac{1}{2}\right)}{\Gamma\left(N+\eta+\frac{1}{2}\right)}{ }_{2} F_{1}\left(\frac{1}{2}, N ; N+\eta+\frac{1}{2} ; a^{2}\right),
$$

where ${ }_{2} F_{1}$ is a hypergeometric function. When differentiating eq. (B.8) with respect to $\eta$ one should keep in mind that for finite $p_{\mathrm{T}}$ the logarithmic derivative of the hypergeometric function is power suppressed in the large- $N$ region.

The $p_{\mathrm{T}}$ distribution at NLO in the threshold limit is finally found to take the form

$$
\frac{d \sigma^{N L O}}{d p_{\mathrm{T}}^{2}}\left(N, p_{\mathrm{T}}^{2}\right)=\frac{\alpha_{s}}{2 \pi} \frac{d \sigma^{L O}}{d p_{\mathrm{T}}^{2}}\left(N, p_{\mathrm{T}}^{2}\right)\left\{c_{2} \ln ^{2}(N)+c_{1}\left(p_{\mathrm{T}}^{2}\right) \ln (N)+O\left(\ln ^{0} N\right)\right\}
$$

with

$$
\begin{aligned}
c_{2} & =3 N_{c}, \\
c_{1}\left(p_{\mathrm{T}}^{2}\right) & =6 \gamma N_{c}-4 N_{c} \ln \left(\frac{Q^{2}}{\mu^{2}}\right)-2 N_{c} \ln \left(\frac{p_{\mathrm{T}}}{Q}\right)+2 \pi b_{0},
\end{aligned}
$$

where $b_{0}$ is given in eq. (A.3). Eqs. (B.10), (B.11) are seen to coincide with the values obtained by expanding the resummed coefficient function to order $\alpha_{s}$, eqs. (4.13), (4.14), since

$$
A_{1}^{g}=C_{A}=N_{c} ; \quad B_{1}^{g}=-\pi b_{0}
$$


Open Access. This article is distributed under the terms of the Creative Commons Attribution License (CC-BY 4.0), which permits any use, distribution and reproduction in any medium, provided the original author(s) and source are credited.

\section{References}

[1] W. Bizoń et al., Fiducial distributions in Higgs and Drell-Yan production at $N^{3} L L+N N L O$, JHEP 12 (2018) 132 [arXiv:1805.05916] [INSPIRE].

[2] W. Bizoń et al., The transverse momentum spectrum of weak gauge bosons at $N^{3} L L+$ NNLO, Eur. Phys. J. C 79 (2019) 868 [arXiv:1905.05171] [inSPIRE].

[3] M.A. Ebert, J.K.L. Michel, I.W. Stewart and F.J. Tackmann, Drell-Yan $q_{T}$ resummation of fiducial power corrections at $N^{3} L L$, JHEP 04 (2021) 102 [arXiv:2006.11382] [INSPIRE].

[4] G. Billis, B. Dehnadi, M.A. Ebert, J.K.L. Michel and F.J. Tackmann, The Higgs $p_{T}$ Spectrum and Total Cross Section with Fiducial Cuts at $N^{3} L L^{\prime}+N^{3} L O$, arXiv:2102.08039 [INSPIRE].

[5] C. Muselli, S. Forte and G. Ridolfi, Combined threshold and transverse momentum resummation for inclusive observables, JHEP 03 (2017) 106 [arXiv:1701.01464] [INSPIRE].

[6] T.R. Rabemananjara, Phenomenology of combined resummation for Higgs and Drell-Yan, JHEP 12 (2020) 073 [arXiv: 2007.09164] [INSPIRE].

[7] M. Bonvini, S. Marzani, C. Muselli and L. Rottoli, On the Higgs cross section at $N^{3} L O+N^{3} L L$ and its uncertainty, JHEP 08 (2016) 105 [arXiv: 1603.08000] [INSPIRE].

[8] D. de Florian, A. Kulesza and W. Vogelsang, Threshold resummation for high-transverse-momentum Higgs production at the LHC, JHEP 02 (2006) 047 [hep-ph/0511205] [INSPIRE].

[9] F.P. Huang, C.S. Li, H.T. Li and J. Wang, Renormalization-group improved predictions for Higgs boson production at large $p_{T}$, Phys. Rev. D 90 (2014) 094024 [arXiv:1406.2591] [INSPIRE].

[10] T. Becher, G. Bell, C. Lorentzen and S. Marti, The transverse-momentum spectrum of Higgs bosons near threshold at NNLO, JHEP 11 (2014) 026 [arXiv:1407.4111] [INSPIRE].

[11] S. Catani, M.L. Mangano and P. Nason, Sudakov resummation for prompt photon production in hadron collisions, JHEP 07 (1998) 024 [hep-ph/9806484] [INSPIRE].

[12] R. Bonciani, S. Catani, M.L. Mangano and P. Nason, Sudakov resummation of multiparton QCD cross-sections, Phys. Lett. B 575 (2003) 268 [hep-ph/0307035] [INSPIRE].

[13] S. Forte and G. Ridolfi, Renormalization group approach to soft gluon resummation, Nucl. Phys. B 650 (2003) 229 [hep-ph/0209154] [INSPIRE].

[14] H. Contopanagos, E. Laenen and G.F. Sterman, Sudakov factorization and resummation, Nucl. Phys. B 484 (1997) 303 [hep-ph/9604313] [INSPIRE].

[15] P. Bolzoni, S. Forte and G. Ridolfi, Renormalization group approach to Sudakov resummation in prompt photon production, Nucl. Phys. B 731 (2005) 85 [hep-ph/0504115] [INSPIRE].

[16] C.J. Glosser and C.R. Schmidt, Next-to-leading corrections to the Higgs boson transverse momentum spectrum in gluon fusion, JHEP 12 (2002) 016 [hep-ph/0209248] [INSPIRE]. 
[17] X. Chen, J. Cruz-Martinez, T. Gehrmann, E.W.N. Glover and M. Jaquier, NNLO QCD corrections to Higgs boson production at large transverse momentum, JHEP 10 (2016) 066 [arXiv: 1607.08817] [INSPIRE].

[18] J.M. Lindert, K. Kudashkin, K. Melnikov and C. Wever, Higgs bosons with large transverse momentum at the LHC, Phys. Lett. B 782 (2018) 210 [arXiv:1801.08226] [INSPIRE].

[19] H.-J. Jing, C.-W. Shen and F.-K. Guo, Graphic Method for Arbitrary n-body Phase Space, Science Bulletin 66 (2021) 653 [arXiv:2005.01942] [INSPIRE].

[20] S. Moch, J.A.M. Vermaseren and A. Vogt, Higher-order corrections in threshold resummation, Nucl. Phys. B 726 (2005) 317 [hep-ph/0506288] [INSPIRE].

[21] E. Gardi and R.G. Roberts, The Interplay between Sudakov resummation, renormalons and higher twist in deep inelastic scattering, Nucl. Phys. B 653 (2003) 227 [hep-ph/0210429] [INSPIRE].

[22] R.D. Ball, M. Bonvini, S. Forte, S. Marzani and G. Ridolfi, Higgs production in gluon fusion beyond NNLO, Nucl. Phys. B 874 (2013) 746 [arXiv:1303.3590] [InSPIRE].

[23] M. Bonvini and S. Marzani, Double resummation for Higgs production, Phys. Rev. Lett. 120 (2018) 202003 [arXiv: 1802.07758] [INSPIRE].

[24] V. Ravindran, J. Smith and W.L. Van Neerven, Next-to-leading order QCD corrections to differential distributions of Higgs boson production in hadron hadron collisions, Nucl. Phys. B 634 (2002) 247 [hep-ph/0201114] [INSPIRE]. 